\title{
Bending rigidity of laminated fabric taking into account the neutral axes of components
}

\author{
KyoungOk Kim ${ }^{1}$, Shigeru Inui ${ }^{2}$, Masayuki Takatera ${ }^{2 *}$ \\ Department of Bioscience and Textile Technology, Interdisciplinary Division of Science and Technology, Shinshu \\ University, Ueda, Japan \\ Faculty of Textile Science and Technology, Shinshu University, Ueda, Japan \\ *takatera@shinshu-u.ac.jp
}

\begin{abstract}
The bending rigidity of laminated fabric was investigated considering the positions of the neutral axes in bending for components in addition to the tensile and in-plane compressive moduli of components. Theoretically derived equations were proposed to obtain the position of the neutral axis and to predict bending rigidity of laminated fabric. Eight face fabrics, ten adhesive interlinings and eighty laminated fabrics of those combinations were used for experimental samples. Tensile properties, bending rigidities and thicknesses of samples were measured and used to investigate the validity of the theory. The positions of the neutral axes for the face fabrics were obtained and they were not close to the centroid of the fabric. The calculated bending rigidities of laminated fabrics using the obtained position of neutral axes were more agreed with the experimental ones than the results by the method without considering the position of neutral axis. Therefore, it was found that the bending rigidity of laminated fabric can be predicted more precisely considering the position of neutral axis.
\end{abstract}

\section{Introduction}

Clothing is made up of a number of subsidiary component fabrics. Among them, interlining is used to give garments a suitable appearance and stability. The interlining is one of the most important subsidiary materials because of its role in the final appearance and function of the garment. An adhesive interlining using a thermoplastic resin is taken as a representative example. The properties of a fabric are considerably changed by laminating on an adhesive interlining. Therefore quantifying the effects of adhesive interlining properties is desirable. Particularly, the prediction of mechanical properties for laminated fabric bonded with adhesive interlining is great interest.

There are some studies about prediction for mechanical properties of laminated fabric made of face fabric and adhesive interlining from the properties of components. Shishoo et al. [1] investigated mechanical properties of laminated fabric with adhesive interlining and analyzed the relationships between mechanical properties of face fabric and adhesive interlining statistically. According to the analyzed relationship, they derived simple regression equations for mechanical properties of laminated fabric. Fan et al. [2] investigated the relationship between the low stress mechanical properties of fused composites and the ones of component fabrics. Based on the relationships, they suggested a set of equations to predict the low stress mechanical properties of fused composites composed of fabric and fusible interlining fabrics. These studies proposed equations of prediction for the mechanical properties of laminated fabric based on statistical analysis. Although the prediction method by statistical analysis is a way of selecting adhesive interlining, a more precise prediction method is necessary because of its insufficient accuracy. Moreover, the relationship between the mechanical properties of adhesive interlining and laminated fabric is still unclear. If it become clear, more precise prediction will be possible.

Among the mechanical properties of laminated fabric, it is well-known that bending rigidity of laminated fabric is much greater than the sum of bending rigidity for each component. Some researchers investigated the prediction for bending rigidity of laminated fabric through the theoretical approaches using the laminate theory of composite structures. Kanayama et al [3 and 4] suggested prediction models for bending rigidity of a composite based on the laminate theory considering the rigidity of adhesive. They verified those models with different type of interlinings. In the laminate theory, the increase amount of bending rigidity is explained as resulting from the strains by compression and extension at the original neutral axes of each component. When a fabric is bent, axial strain does not arise in the neutral axis. However, due to the laminating, strains by an extension and a compression of the original neutral axes will arise. Thus, the strains by these extension and compression of the neutral axis form stress so that the bending rigidity of a laminated fabric will increase as the amount of the moment by the resultant force. If the elastic moduli in tensile and bending are the same, bending rigidity of laminated fabric per unit breadth is given by Equation (1):

$$
B_{12}=3 B_{1} B_{2} \frac{\left(h_{1}+h_{2}\right)^{2}}{\left(B_{1} h_{2}^{2}+B_{2} h_{1}^{2}\right)}+B_{1}+B_{2} \text {, }
$$

where $B_{12}, B_{1}$ and $B_{2}$ are bending rigidities per unit breadth of laminated fabric, adhesive interlining and face fabric, respectively. $h_{1}$ and $h_{2}$ are the thicknesses of adhesive interlining and face fabric. Kim et al [5] verified Equation (1) taking into account the change on the mechanical properties of face fabric and adhesive interlining by press used in the laminating process. 
In Equation (1), it was assumed that the components are perfectly elastic continua. However, textile materials are not elastic continua, so the bending rigidity of laminate fabric has been separated into two parts, the contributions from the bending of the components and their extension and compression [6]. This is equivalent to differentiating between the tensile and bending moduli of the fabrics. Those differences have an effect on the bending rigidity of laminated fabric. If the elastic modulus in bending and tensile of both fabrics are independent and the neutral axes in bending are assumed to lie in the centroid of the cross section, the bending rigidity of laminated fabric is given by

$$
B_{12}=\frac{T_{2 T} T_{1 C}}{T_{1 C}+T_{2 T}} \cdot\left(\frac{h_{2}+h_{1}}{2}\right)^{2}+B_{1}+B_{2}
$$

where $T_{1 C}$ and $T_{2 T}$ are the apparent in-plane compressive modulus of adhesive interlining and apparent tensile modulus of face fabric assumed to be a constant. Kim et al. [7] proposed a method to obtain $T_{1 C}$ from laminated fabric with the different combinations of face fabric and adhesive interlining. Using the obtained $T_{1 C}$, they confirmed the validity of Equation (2). In the predicted results by Equation (2), most of the predicted data were close to the experimental ones than the ones by Equation (1).

However, some fabrics still showed relatively larger prediction errors than other fabrics. In this study, the reason of the errors was considered in detail. In Equation (2), the neutral axes of components were assumed to lie in the centroids. If the assumption is invalid, the bending rigidity of laminated fabric will be affected by it and the errors may be shown. Accordingly, it will be necessary to understand the effect of the position of neutral axes in components on the laminate bending rigidity.

In this study, a detailed bending theory of laminated fabric was proposed taking into account the position of neutral axes of fabrics in components. The theoretical equations of bending rigidity of laminated fabric were verified experimentally with samples which showed relatively large prediction errors by Equation (2).

\section{Theoretical}

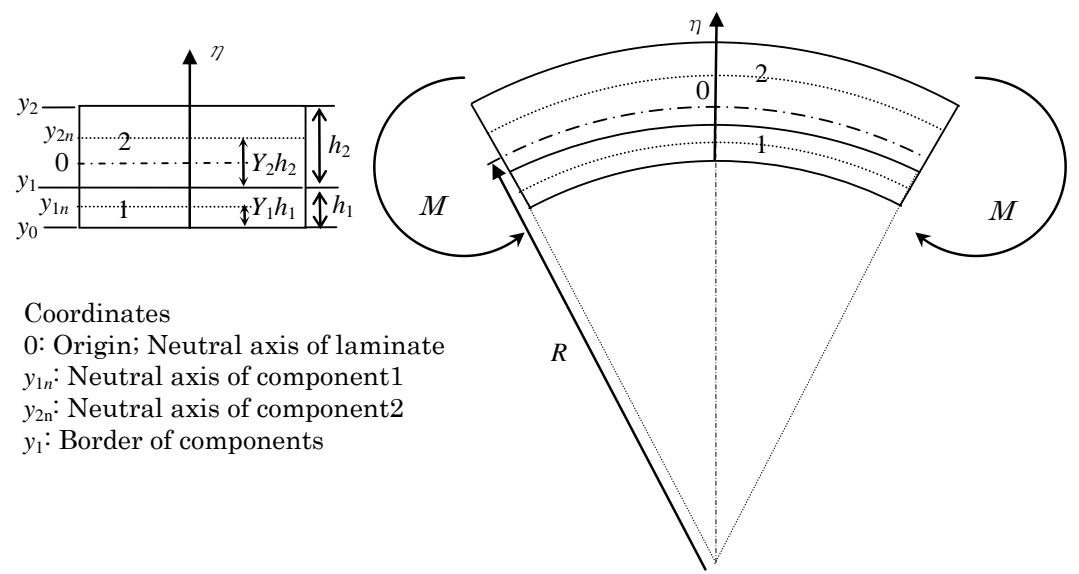

Figure 1 Bending of two-component laminate

Let us consider a laminated fabric with component 1 and component 2 which neutral axes before bonding do not pass through the centroid. A cross section through a laminated fabric, bent to a radius of curvature $R$ is shown in Figure 1. In Figure 1, component 1 is inward and component 2 is outward. $\eta$ is the distance from the neutral surface in the laminated fabric. We take the origin at the neutral axis of the laminate. $y_{0}$ and $y_{1}, y_{2}$ are the coordinates of surface and boundaries. $y_{1 n}$ and $y_{2 n}$ are the coordinates of the original neutral axes of components. Because the strain of each component is proportional to the distance from the origin, the component 1 is compressed and the component 2 is extended while bending. It was assumed that a linear stress and strain relation is valid. Assuming the strains by extension and compression at the neutral axes of components are the mean strain in component, a couple of forces will be occurred due to the stresses by the strains.

The compressive strain at the neutral axis of component $1, \varepsilon_{1}$ before bonding is given by

$\varepsilon_{1}=\frac{y_{1 n}}{R}$

Then, the stress in component $1, \sigma_{1}$ is given by:

$\sigma_{1}=\frac{y_{1 n} E_{1 C}}{R}$

where $E_{1 C}$ is the compressive modulus of component1. The total force of component1, $F_{1}$ is

$F_{1}=\frac{y_{1 n} E_{1 C} h_{1} b}{R}$ 
where $b$ is the breadth of each component.

Similarly, the total force of component $2, F_{2}$ is

$F_{2}=\frac{y_{2 n} E_{2 T} h_{2} b}{R}$

where $E_{2 T}$ is the tensile modulus of component 2.

In experiments with fabrics, elastic modulus per breadth is usually used for convenience. Thus, specified moduli $T_{1 C}$ and $T_{2 T}$ are introduced as follows.

$T_{1 C} \equiv E_{1 C} h_{1}, T_{2 T} \equiv E_{2 C} h_{2}$

For no external axial force, the sum of two resultant forces in Equations (5) and (6) should be zero.

$y_{1 n} T_{1 C}+y_{2 n} T_{2 T}=0$

When the distances from the bottom to the neutral axis of each component are denoted by $y_{1 n 0}$ and $y_{2 n 0}$, the relative positions $Y_{1}$ and $Y_{2}$ are expressed as follows:

$Y_{1}=y_{1 n 0} / h_{1}$

$Y_{2}=y_{2 n 0} / h_{2}$

Then $y_{1 n}$ and $y_{2 n}$ are expressed as follows:

$y_{1 n}=y_{1}-h_{1}\left(1-Y_{1}\right)$

$y_{2 n}=y_{1}+Y_{2} h_{2}$

Substituting Equations (12) into (8), we can obtain

$T_{1 C}\left(y_{1}-h_{1}\left(1-Y_{1}\right)\right)+T_{2 T}\left(y_{1}+Y_{2} h_{2}\right)=0$

Then, $y_{1}$ is as follows:

$y_{1}=\frac{T_{1 C} h_{1}\left(1-Y_{1}\right)-T_{2 T} Y_{2} h_{2}}{T_{1 C}+T_{2 T}}$

That gives the neutral axis of the laminate. Substituting Equation (14) into (12), $y_{2 n}$ can be obtained:

$y_{2 n}=\frac{T_{1 C}\left\{h_{1}\left(1-Y_{1}\right)+Y_{2} h_{2}\right\}}{T_{1 C}+T_{2 T}}$

From Equation (8), we can obtain $y_{1 n}$ as follows:

$y_{1 n}=-y_{2 n} \frac{T_{2 T}}{T_{1 C}}=-\frac{T_{2 T}\left\{h_{1}\left(1-Y_{1}\right)+Y_{2} h_{2}\right\}}{T_{1 C}+T_{2 T}}$

Because the two forces, Equations (5) and (6), are equal and opposite, they form a couple. By the assumption, the forces act at the centroid of components, and then the distance between the forces is $\left(h_{2}+h_{1}\right) / 2$. Accordingly, the couple per unit breadth and unit curvature, $M_{1}$ and $M_{2}$ is

$M_{1}=-y_{1 n} T_{1 C} \cdot \frac{\left(h_{1}+h_{2}\right)}{2}$

or

$M_{2}=y_{2 n} T_{2 T} \cdot \frac{\left(h_{1}+h_{2}\right)}{2}$

This is the contribution from extension and compression of components. The bending rigidity of laminated fabric is given by the sum of these contributions and the contributions from the bending of the components.

Therefore, the bending rigidity of a laminated fabric can be expressed as follows:

$B_{12}=-T_{1 C} y_{1 n} \cdot \frac{\left(h_{1}+h_{2}\right)}{2}+B_{1}+B_{2}$

or

$B_{12}=T_{2 T} y_{2 n} \cdot \frac{\left(h_{1}+h_{2}\right)}{2}+B_{1}+B_{2}$

By substituting Equation (15) into (20), we can finally express bending rigidity of the laminated fabric as follows:

$B_{12}=\frac{T_{1 C} T_{2 T}\left(\left(1-Y_{1}\right) h_{1}+Y_{2} h_{2}\right)\left(h_{1}+h_{2}\right)}{2\left(T_{1 C}+T_{2 T}\right)}+B_{1}+B_{2}$

Bending rigidity of laminated fabric with two components which neutral axes do not exist on those centroids can be predicted with Equation (21). However, among the parameters, $T_{1 C}, Y_{1}$ and $Y_{2}$ cannot be measured directly [8]. Therefore, a method to obtain those values was considered from the experiments under a specific assumption.

If $Y_{1}=1 / 2$, Equation (21) can be expressed as follows:

$B_{12}=\frac{T_{2 T} T_{1 C}\left(h_{1}+2 Y_{2} h_{2}\right)\left(h_{2}+h_{1}\right)}{4\left(T_{1 C}+T_{2 T}\right)}+B_{1}+B_{2}$ 
On the other hand, if $Y_{2}=1 / 2$, we have

$B_{12}=\frac{T_{1 C} T_{2 T}\left(2\left(1-Y_{1}\right) h_{1}+h_{2}\right)\left(h_{1}+h_{2}\right)}{4\left(T_{1 C}+T_{2 T}\right)}+B_{1}+B_{2}$

Furthermore, if $Y_{1}=Y_{2}=1 / 2$, we obtain Equation (23) and it is the same with Equation (2).

$B_{12}=\frac{T_{2 T} T_{1 C}\left(h_{2}+h_{1}\right)^{2}}{4\left(T_{1 C}+T_{2 T}\right)}+B_{1}+B_{2}$

Let us consider the position of a neutral axis of a fabric theoretically. As has already been stated, the position of the neutral axis for a bent component cannot be measured experimentally. If the $E_{B T}$ and $E_{B C}$, which are tensile and compressive moduli in bending, are known, the relative position, $Y$ of the neutral axis can be theoretically given by

$Y=\frac{\sqrt{E_{B T}}}{\sqrt{E_{B C}}+\sqrt{E_{B T}}}$

then the bending rigidity, $B$ of the fabric is expressed as follows[8].

$B=\frac{E_{B T} E_{B C} h^{3}}{3\left(\sqrt{E_{B C}}+\sqrt{E_{B T}}\right)^{2}}=\frac{Y^{2} h^{3} E_{B C}}{3}=\frac{(1-Y)^{2} h^{3} E_{B T}}{3}$

However, $E_{B C}, E_{B T}$ and $Y$ are unknown and cannot be measured directly. Therefore, an indirect approach was considered to obtain the necessary parameters with laminated fabrics in this study.

Firstly, let us consider Equation (22). If $Y_{1}=1 / 2$, bending rigidity of laminated fabric can be predicted using Equation (22) with $B_{1}, B_{2}, T_{1 C}, T_{2 T}, h_{1}, h_{2}$ and $Y_{2}$. In here, $B_{1}$ and $B_{2}$ can be measured by a pure bending test. $h_{1}$ and $h_{2}$ can be measured. $T_{2 T}$ can be measured by a tensile test. $T_{1 C}$ is apparent compressive modulus of component 1 and there are some studies about measuring it [9 and 10]. However those studies only suggested some possibilities of the measurement and a reliable method was not established. It is still difficult to measure it directly. Instead of a direct measuring method, indirect method can be used. From Equation (23), $T_{1 C}$ can be expressed as Equation (26), and then $T_{1 C}$ can be obtained.

$T_{1 C}=\frac{\left(B_{12}-B_{1}-B_{2}\right) T_{2 T}}{\left(B_{12}-B_{1}-B_{2}\right)-T_{2 T}\left(\frac{h_{2}+h_{1}}{2}\right)^{2}}=\frac{1}{\frac{1}{T_{2 T}}-\frac{\left(h_{2}+h_{1}\right)^{2}}{4\left(B_{12}-B_{1}-B_{2}\right)}}$

Using Equations (23) and (26), Kim et al. [7] predicted the bending rigidities of laminated fabrics with face fabrics and adhesive interlinings with an assumption that those neutral axes lie in those centroids. In the study, with $T_{1 C}$ from two twill fabrics using Equation (26), the predicted bending rigidities of laminated fabrics with adhesive interlinings showed good agreement with experimental ones. Thus, the assumption that the neutral axes of the twill face fabrics pass through the centroid was verified, and thus the $T_{1 C}$ can be used in Equation (22) as well.

If $T_{1 C}$ is obtained, $Y_{2}$ can be obtained with the following equation:

$Y_{2}=\frac{2\left(B_{12}-B_{1}-B_{2}\right)}{h_{2}\left(h_{1}+h_{2}\right)}\left(\frac{1}{T_{2 T}}+\frac{1}{T_{1 C}}\right)-\frac{h_{1}}{2 h_{2}}$

Therefore, when the neutral axis of component 1 is assumed to lie in the centroid, bending rigidity of laminated fabric with component 2 which neutral axis is unknown can be predicted using Equation (22) with $T_{1 C}, h_{1}$ and $B_{1}$ of component 1 and $T_{2 T}, h_{2}, B_{2}$ and $Y_{2}$ of component 2 obtained by Equation (27).

Let us consider Equation (22)'. Equation (22)' can be applied to a reverse bending of Equation (22) that component 1 is outward and the component 2 is inward in bending. In Equation (22)', $T_{2 C}$ of a component 2 which neutral axis does not lie in the centroid will be necessary. However, the measuring method of $T_{2 C}$ for the component 2 has not been reported yet. To verify the prediction of laminated fabric in the case of Equation (22)', further study on the obtaining method of $T_{2 C}$ in the case that the neutral axis does not exist in the centroid will be necessary in the future.

In summary, the bending rigidity of laminated fabric can be predicted with four moduli $\left(T_{T}, T_{\mathrm{C}}, E_{B T}\right.$ and $\left.E_{B C}\right)$ or the relative position of neutral axis, $Y$ instead of $E_{B T}$ and $E_{B C}$ for component fabrics by the proposed method. For the reverse direction bending, due to the different neutral axis on the bending direction, the other relative position of the neutral axis $Y^{\prime}$ will be necessary.

\section{Experimental}

Experiments were carried out to verify the proposed equations for predicting the bending rigidity of laminated fabric from mechanical properties of face fabric and adhesive interlining before bonding. In this study, the bending rigidity of a laminated fabric was considered except for the stiffness properties of a lining. In the usage of adhesive interlinings, an adhesive interlining is usually used on the inward of clothing and the face fabric was on the outward of the arc of bending. Thus, Equation (22) was verified by assuming component 1 for an interlining and component 2 for a face fabric.

Face fabrics, adhesive interlinings and laminated fabrics with those combinations were prepared as experimental samples. Bending rigidities on warp and weft direction respectively of all samples were measured using a 
KES-FB2 pure bending tester [11]. The thickness of each sample was measured using a KES-FB3 compression tester at $49 \mathrm{~Pa}$ load. The tensile properties of samples were measured by KES-FB1 tensile tester up to a maximum load of 490 $\mathrm{cN} / \mathrm{cm}$. The load at 0 to about $2.5 \%$ of elongation from the load-elongation curve was used to calculate the $T_{2 T}$ for each face fabric [7]. Every test was carried out under standard conditions $\left(20 \pm 1^{\circ} \mathrm{C}\right.$ and $65 \pm 5 \%$ relative humidity). All samples were preconditioned under these standard conditions for 24 hours. Every test was conducted on five samples and the results were averaged.

The $T_{1 \mathrm{C}}$ was calculated by Equation 26 with values obtained by the experiment in the combination of specific face fabric and interlining. Then $Y_{2}$ of face fabrics were calculated by Equation (27). Using the $Y_{2}$ values, the bending rigidities of other laminated fabrics bonded with the face fabrics and different interlinings were predicted with Equation (22). Those results were compared with experimental data.

The sample specifications are shown in tables 1,2 and 3. Particularly, we used face fabrics which showed large prediction errors by Equation (2). Hence, six fabrics (S1-6) which showed large prediction errors (over about mean absolute percentage errors (MAPE) 10\%) using Equation (2) were prepared as face fabric samples assumed to have different tensile and compressive moduli. Ten kinds of adhesive interlinings (CE1-5 and DP1-5) were also prepared as experimental samples. Two twill fabrics (N1 and N2), which neutral axes can be assumed to lie in the centroid [7], were prepared to obtain the compressive moduli of adhesive interlinings. Eighty combinations of laminated fabrics were constructed and examined. Bonding interlining to face fabric was done automatically using a press machine (Kobe Denki Kogyosyo, BP-V4812D). The bonding conditions were $150^{\circ} \mathrm{C}$, under $29.4 \mathrm{kPa}$ load and 10 s pressing time.

The mechanical properties of component fabrics were changed after pressing procedure for laminating and the changes of the mechanical properties for laminated fabrics must be considered when predicting the bending rigidity of laminated fabrics [5]. Therefore, samples pressed alone under the same press conditions of laminating and those mechanical properties were measured. The manufacturing method is as follows: Face fabric samples were pressed under the same conditions as bonding interlining. To press the adhesive interlining, polytetrafluoroethylene (PTFE) film (NITTO, No.900, 0.05 $\times 300 \mathrm{~mm}$ ) was prepared. Adhesive interlinings were bonded to PTFE films and PTFE films were removed from adhesive interlining. The samples pressed alone were referred to as 'pressed samples', and face fabric and adhesive interlining together as 'pressed adhesive interlining' and 'pressed samples'. The conditions for manufacturing the pressed samples were the same as for bonding interlining.

Table 1 Specifications of the face fabrics

\begin{tabular}{|c|c|c|c|c|c|c|}
\hline \multirow{2}{*}{$\begin{array}{l}\text { Sample } \\
\text { name }\end{array}$} & \multicolumn{2}{|c|}{ Yarn Count(Nm) } & \multirow[t]{2}{*}{ Weave } & \multirow{2}{*}{$\begin{array}{c}\operatorname{Density}(/ \mathrm{cm}) \\
(\text { Warp } \times \text { Weft })\end{array}$} & \multirow[t]{2}{*}{ Material } & \multirow{2}{*}{$\begin{array}{l}\text { Pressed face } \\
\text { fabric name }\end{array}$} \\
\hline & Warp & Weft & & & & \\
\hline N1 & $\begin{array}{c}16.5 \text { tex } \times 2 ; \\
\text { R33tex }\end{array}$ & $\begin{array}{c}16.5 \text { tex } \times 2 ; \\
\text { R33tex }\end{array}$ & Twill & $28 \times 22$ & Wool 100\% & P-N1 \\
\hline $\mathrm{N} 2$ & $\begin{array}{c}14 \text { tex } \times 2 \\
\text { R28tex }\end{array}$ & $\begin{array}{c}14 \text { tex } \times 2 \\
\text { R28tex }\end{array}$ & Twill & $29 \times 24$ & Wool 100\% & $\mathrm{P}-\mathrm{N} 2$ \\
\hline $\mathrm{S} 1$ & $\begin{array}{l}\text { 60tex } \times 2 ; \\
\text { R 120tex }\end{array}$ & 30tex & Satin & $50 \times 40$ & Wool100\% & P-S1 \\
\hline $\mathrm{S} 2$ & $\begin{array}{l}\text { 42tex } \times 2 \\
\text { R84tex }\end{array}$ & 30tex & Satin & $45 \times 33$ & Wool100\% & P-S2 \\
\hline $\mathrm{S} 3$ & 47tex & 36 tex & Satin & $54 \times 37$ & $\mathrm{~W} 75 \%, \mathrm{P} 25 \%$ & P-S3 \\
\hline $\mathrm{S} 4$ & 36tex & 30tex & Satin & $53 \times 37$ & $\mathrm{~W} 75 \%, \mathrm{P} 25 \%$ & P-S4 \\
\hline S5 & $\begin{array}{l}\text { 47tex } \times 2 ; \\
\text { R94tex }\end{array}$ & 30 tex & Satin & $42 \times 30$ & Wool100\% & P-S5 \\
\hline S6 & $\begin{array}{l}14 \text { tex } \times 2 ; \\
\text { R28tex }\end{array}$ & $\begin{array}{l}14 \text { tex } \times 2 ; \\
\text { R28tex }\end{array}$ & Satin & $43 \times 29$ & Wool $85 \%$, Angora $15 \%$ & P-S6 \\
\hline
\end{tabular}

Table 2 Specifications of the adhesive interlinings

\begin{tabular}{|c|c|c|c|c|c|c|}
\hline $\begin{array}{l}\text { Sample } \\
\text { name }\end{array}$ & $\begin{array}{l}\text { Density } \\
\text { (/cm) }\end{array}$ & $\begin{array}{l}\text { Adhesive dot } \\
\text { number }(/ \mathrm{cm}) \\
(\text { warp } \times \text { weft })\end{array}$ & $\begin{array}{l}\text { Adhesive } \\
\text { dot } \\
\text { size }(\mathbf{m m})\end{array}$ & $\begin{array}{l}\text { Mass per unit } \\
\operatorname{area}\left(g / \mathbf{m}^{2}\right)\end{array}$ & $\begin{array}{c}\text { Adhesive mass } \\
\text { without } \\
\text { fabric }\left(\mathrm{g} / \mathbf{m}^{2}\right)\end{array}$ & $\begin{array}{l}\text { Pressed adhesive } \\
\text { interlining name }\end{array}$ \\
\hline CE1 & $38 \times 22$ & $10 \times 10$ & 0.17 & 36.2 & 8.6 & P-CE-1 \\
\hline CE2 & $38 \times 23$ & $10 \times 10$ & 0.17 & 35.6 & 8.0 & P-CE-2 \\
\hline CE3 & $38 \times 25$ & $10 \times 10$ & 0.17 & 36.5 & 8.3 & P-CE-3 \\
\hline CE4 & $37 \times 26$ & $10 \times 10$ & 0.17 & 36.5 & 8.1 & P-CE-4 \\
\hline CE5 & $37 \times 26$ & $10 \times 10$ & 0.17 & 35.7 & 7.7 & P-CE-5 \\
\hline DP1 & $39 \times 24$ & $9 \times 9$ & 0.25 & 38.5 & 8.7 & P-DP-1 \\
\hline DP2 & $39 \times 24$ & $10 \times 10$ & 0.23 & 39.9 & 10.0 & P-DP-2 \\
\hline DP3 & $39 \times 24$ & $10 \times 10$ & 0.30 & 41.8 & 11.6 & P-DP-3 \\
\hline DP4 & $39 \times 24$ & $11 \times 11$ & 0.20 & 37.5 & 8.7 & P-DP-4 \\
\hline DP5 & $39 \times 24$ & $12 \times 12$ & 0.10 & 39.3 & 10.1 & P-DP-5 \\
\hline
\end{tabular}


Table 3 Combinations of face fabric and adhesive interlining

\begin{tabular}{|c|c|c|c|c|c|c|c|c|}
\hline $\begin{array}{l}\text { Face fabric } \\
\text { Adhesive interlining }\end{array}$ & N1 & $\mathbf{N 2}$ & S1 & $\mathbf{S 2}$ & $\mathbf{S 3}$ & S4 & S5 & S6 \\
\hline CE1 & $\mathrm{N} 1-\mathrm{CE} 1$ & N2-CE1 & A-CE1 & S2-CE1 & S3-CE1 & S4-CE1 & S5-CE1 & S6-CE1 \\
\hline CE2 & N1-CE2 & $\mathrm{N} 2-\mathrm{CE} 2$ & A-CE2 & S2-CE2 & S3-CE2 & S4-CE2 & S5-CE2 & S6-CE2 \\
\hline CE3 & N1-CE3 & $\mathrm{N} 2-\mathrm{CE} 3$ & A-CE3 & $\mathrm{S} 2-\mathrm{CE} 3$ & S3-CE3 & S4-CE3 & S5-CE3 & S6-CE3 \\
\hline CE4 & $\mathrm{N} 1-\mathrm{CE} 4$ & $\mathrm{~N} 2-\mathrm{CE} 4$ & A-CE4 & $\mathrm{S} 2-\mathrm{CE} 4$ & S3-CE4 & S4-CE4 & S5-CE4 & S6-CE4 \\
\hline CE5 & N1-CE5 & N2-CE5 & A-CE5 & S2-CE5 & S3-CE5 & S4-CE5 & S5-CE5 & S6-CE5 \\
\hline DP1 & N1-DP1 & N2-DP1 & A-DP1 & S2-DP1 & S3-DP1 & S4-DP1 & S5-DP1 & S6-DP1 \\
\hline DP2 & N1-DP2 & N2-DP2 & A-DP2 & S2-DP2 & S3-DP2 & S4-DP2 & S5-DP2 & S6-DP2 \\
\hline DP3 & N1-DP3 & N2-DP3 & A-DP3 & S2-DP3 & S3-DP3 & S4-DP3 & S5-DP3 & S6-DP3 \\
\hline DP4 & N1-DP4 & N2-DP4 & A-DP4 & S2-DP4 & S3-DP4 & S4-DP4 & S5-DP4 & S6-DP4 \\
\hline DP5 & N1-DP5 & N2-DP5 & A-DP5 & S2-DP5 & S3-DP5 & S4-DP5 & S5-DP5 & S6-DP5 \\
\hline
\end{tabular}

\section{Results and Discussions}

The bending rigidities and thicknesses of pressed samples are shown in tables 4 and 5. Bending rigidities which were taken when the face fabric was on the outside of the arc in bending were used because an adhesive interlining is usually used on the inside of the arc in bending of clothing. The $T_{2 T}$ values from the tensile properties of pressed face fabrics are shown in table 6. $T_{1 C}$ values for adhesive interlining samples were obtained using Equation (26) from the laminated fabric with twill fabrics $\mathrm{N} 1$ and $\mathrm{N} 2$. The averages of $T_{1 C}$ values were obtained as shown in Table 7 and used in the prediction.

To predict bending rigidity of laminated fabric with a face fabric which the neutral axis does not pass through the centroid, $Y_{2}$ of face fabric is necessary. With the obtained bending rigidity, thickness and tensile and compressive moduli, $Y_{2}$ of face fabrics were calculated by Equation (27) and the averages for all samples were shown in Figure 2. $Y_{2}$ values of the face fabrics were similar for different adhesive interlinings. Thus, it was verified that the position of the neutral axis for the face fabric can be obtained by Equation (27). As shown in Figure 2, the averages of $Y_{2}$ for all samples were closed to 1 . If $Y_{2}$ is close to 0.5 , it means that $E_{2 C}$ and $E_{2 T}$ are almost the same. In that case, the predicted results will be similar with the results by Equation (2). On the other hand, if $Y_{2}$ is close to 1, it means that $E_{2 C}$ and $E_{2 T}$ are very different. In that case, the neutral axis of the face fabric is close to the top of face fabric. Therefore, it was confirmed that the neutral axes of the face fabrics (S1-6) which showed large prediction errors by Equation (2) do not lie close to the centroid.

Although the $Y_{2}$ values of the face fabrics showed similar ones for different adhesive interlinings, some variations were still shown between samples. The reasons will be the permeation of adhesive agent on face fabric and nonlinear properties of fabrics. However, the variations were not so large that the values can be acceptable.

If the predicted bending rigidities of laminated fabric with the obtained $Y_{2}$ are agreed with experimental ones, it means that the obtained $Y_{2}$ values are reasonably valid and can be used to predict bending rigidity of laminated fabric with other combinations. Thus, the bending rigidities of the laminated fabrics with other interlinings were predicted with the obtained $Y_{2}$. In here, $Y_{2}$ values of face fabric from laminated fabric with CE4 interlining were used because those showed the closest values to the averages of the obtained $Y_{2}$ values as shown in Figure 2. Figure 3 shows the comparison of predicted and experimental bending rigidities for laminated fabrics using $Y_{2}$. The predicted bending rigidities using Equation (2) are also shown in Figure 3 to confirm the effect of considering the position of neutral axis. The MAPE from the results by the method considered $Y_{2}$ and without considering $Y_{2}$ are shown in Table 8. As shown in Figure 3 and in its MAPE, the predicted bending rigidities with the obtained $Y_{2}$ showed closer agreement with the experimental data than those from method without considering $Y_{2}$. The reason of the better agreements is the position of the neutral axes of composites. Because a fabric is not elastic continua, the assumption that the neutral axis exists in the centroid is not always valid. These results showed that the bending rigidity of a laminated fabric was significantly influenced by the position. The position is necessary to be considered especially for the bending rigidity of a laminated fabric. Thus, the predicted bending rigidities of laminated fabric considered $Y_{2}$ were more agreed with the experimental ones than the predicted ones without considering $Y_{2}$. It became clear that the bending rigidities of laminated fabric were able to be predicted more precisely with the method considered $Y_{2}$ than using method without considering it. MAPE of all samples from the method considered $Y_{2}(3.6 \%)$ was less than the results by method without considering $Y_{2}(12.7 \%)$. This was a valuable improvement so it became clear that the concerning position of the neutral axis is meaningful. Although the predicted bending rigidities of all samples showed better agreements, the MAPE is different depending on samples as shown in Table 8. The reason will be due to the variation of $Y_{2}$. 
Table 4 Bending rigidities and thicknesses of pressed interlinings

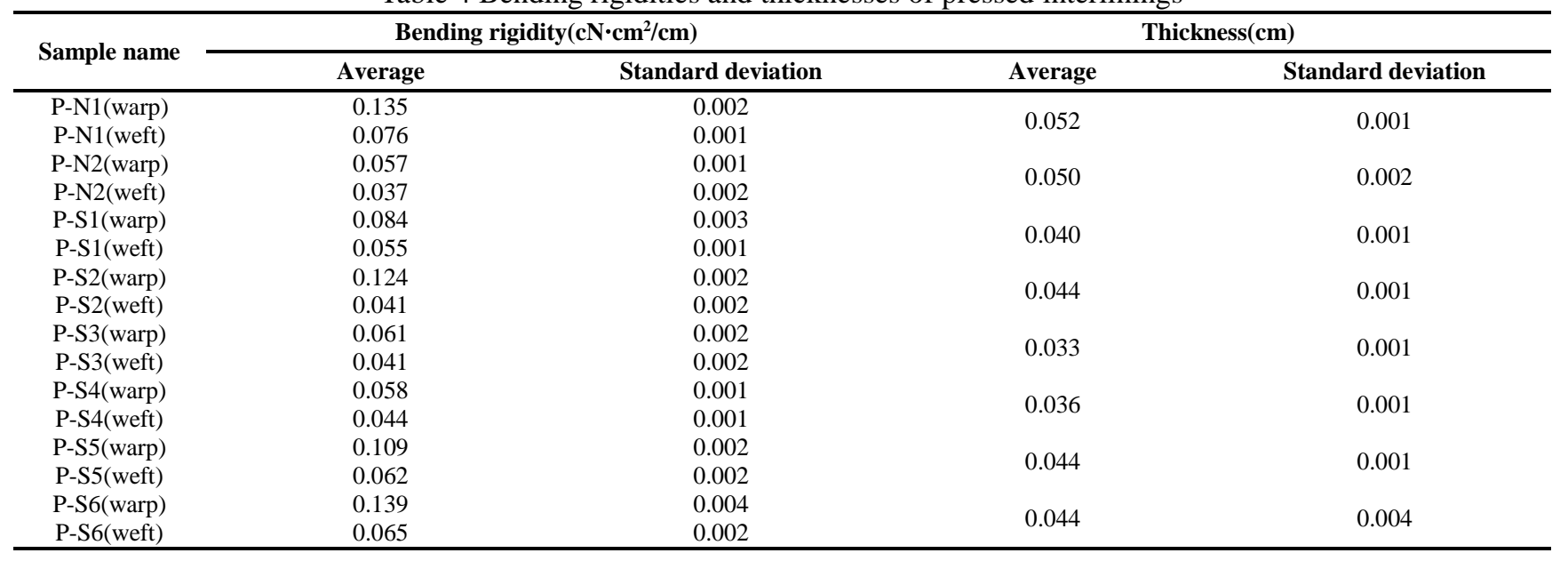

Table 5 Bending rigidities and thicknesses of pressed interlinings

\begin{tabular}{|c|c|c|c|c|c|c|c|c|c|}
\hline \multirow{2}{*}{ Sample name } & \multicolumn{2}{|c|}{$\begin{array}{c}\text { Bending rigidity } \\
\left(\mathrm{cN} \cdot \mathrm{cm}^{2} / \mathrm{cm}\right)\end{array}$} & \multicolumn{2}{|c|}{$\begin{array}{c}\text { Thickness } \\
(\mathbf{c m})\end{array}$} & \multirow{2}{*}{ Sample name } & \multicolumn{2}{|c|}{$\begin{array}{c}\text { Bending rigidity } \\
\left(\mathrm{cN} \cdot \mathrm{cm}^{2} / \mathrm{cm}\right)\end{array}$} & \multicolumn{2}{|c|}{$\begin{array}{c}\text { Thickness } \\
\text { (cm) }\end{array}$} \\
\hline & Average & $\begin{array}{l}\text { Standard } \\
\text { deviation }\end{array}$ & Average & $\begin{array}{l}\text { Standard } \\
\text { deviation }\end{array}$ & & Average & $\begin{array}{l}\text { Standard } \\
\text { deviation }\end{array}$ & Average & $\begin{array}{l}\text { Standard } \\
\text { deviation }\end{array}$ \\
\hline P-CE1(warp) & 0.0058 & 0.0015 & \multirow{2}{*}{0.027} & \multirow{2}{*}{0.001} & P-DP1(warp) & 0.0064 & 0.0009 & \multirow{2}{*}{0.024} & \multirow{2}{*}{0.007} \\
\hline P-CE1(weft) & 0.0051 & 0.0016 & & & P-DP1(weft) & 0.0024 & 0.0010 & & \\
\hline P-CE2(warp) & 0.0058 & 0.0016 & \multirow{2}{*}{0.025} & \multirow{2}{*}{0.001} & P-DP2(warp) & 0.0059 & 0.0011 & \multirow{2}{*}{0.024} & \multirow{2}{*}{0.006} \\
\hline P-CE2(weft) & 0.0030 & 0.0010 & & & P-DP2(weft) & 0.0020 & 0.0013 & & \\
\hline P-CE3(warp) & 0.0060 & 0.0005 & \multirow{2}{*}{0.024} & \multirow{2}{*}{0.001} & P-DP3(warp) & 0.0074 & 0.0007 & \multirow{2}{*}{0.025} & \multirow[t]{2}{*}{0.007} \\
\hline P-CE3(weft) & 0.0035 & 0.0009 & & & P-DP3(weft) & 0.0033 & 0.0009 & & \\
\hline P-CE4(warp) & 0.0070 & 0.0005 & \multirow{2}{*}{0.024} & \multirow{2}{*}{0.0004} & P-DP4(warp) & 0.0059 & 0.0008 & \multirow{2}{*}{0.024} & \multirow{2}{*}{0.007} \\
\hline P-CE4(weft) & 0.0039 & 0.0005 & & & P-DP4(weft) & 0.0027 & 0.0008 & & \\
\hline P-CE5(warp) & 0.0085 & 0.0033 & \multirow{2}{*}{0.023} & \multirow{2}{*}{0.001} & P-DP5(warp) & 0.0062 & 0.0008 & \multirow{2}{*}{0.025} & \multirow{2}{*}{0.007} \\
\hline P-CE5(weft) & 0.0039 & 0.0013 & & & P-DP5(weft) & 0.0013 & 0.0017 & & \\
\hline
\end{tabular}

Table $6 T_{2}$ values of pressed face fabrics

\begin{tabular}{cc}
\hline Sample name & $\boldsymbol{T}_{2}$ values(N/cm) \\
\hline P-A(warp) & 14.7 \\
P-A(weft) & 29.5 \\
P-B(warp) & 23.3 \\
P-B(weft) & 4.3 \\
P-S1(warp) & 20.9 \\
P-S21weft) & 43.7 \\
P-S2(warp) & 125 \\
P-S2(weft) & 26.0 \\
P-S3(warp) & 51.8 \\
P-S3(weft) & 39.5 \\
P-S4(warp) & 61.3 \\
P-S4(weft) & 56.6 \\
P-S5(warp) & 56.3 \\
P-S6(warp) & 114 \\
P-S6(weft) & 13.3 \\
\hline
\end{tabular}

Table 7 Average $T_{1}$ values of pressed interlinings from face fabric, N1and N2

\begin{tabular}{cccc}
\hline Sample name & $\boldsymbol{T}_{\boldsymbol{I}}$ values(N/cm) & Sample name & $\boldsymbol{T}_{\boldsymbol{I}}$ values(N/cm) \\
\hline P-CE1(warp) & 0.69 & P-DP1(warp) & P-DP1(weft) \\
P-CE1(weft) & 0.39 & P-DP2(warp) & 0.18 \\
P-CE2(warp) & 0.78 & P-DP2(weft) & 0.78 \\
P-CE2(weft) & 0.39 & P-DP3(warp) & 0.39 \\
P-CE3(warp) & 0.78 & P-DP3(weft) & 1.27 \\
P-CE3(weft) & 0.39 & P-DP4(warp) & 0.39 \\
P-CE4(warp) & 0.88 & P-DP4(weft) & 1.27 \\
P-CE4(weft) & 0.39 & P-DP5(warp) & 0.29 \\
P-CE5(warp) & 0.98 & P-DP5(weft) & 1.18 \\
P-CE5(weft) & 0.39 & & 0.29 \\
\hline
\end{tabular}




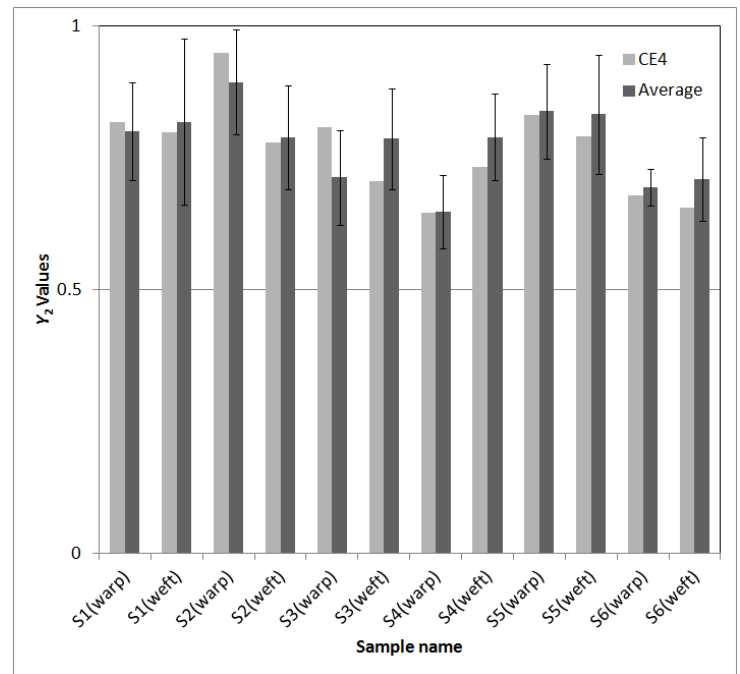

Figure 2 Averages of $Y_{2}$ values of face fabrics for different adhesive interlinings and $Y_{2}$ values from laminated fabric combinations of face fabrics and CE4 interlining.

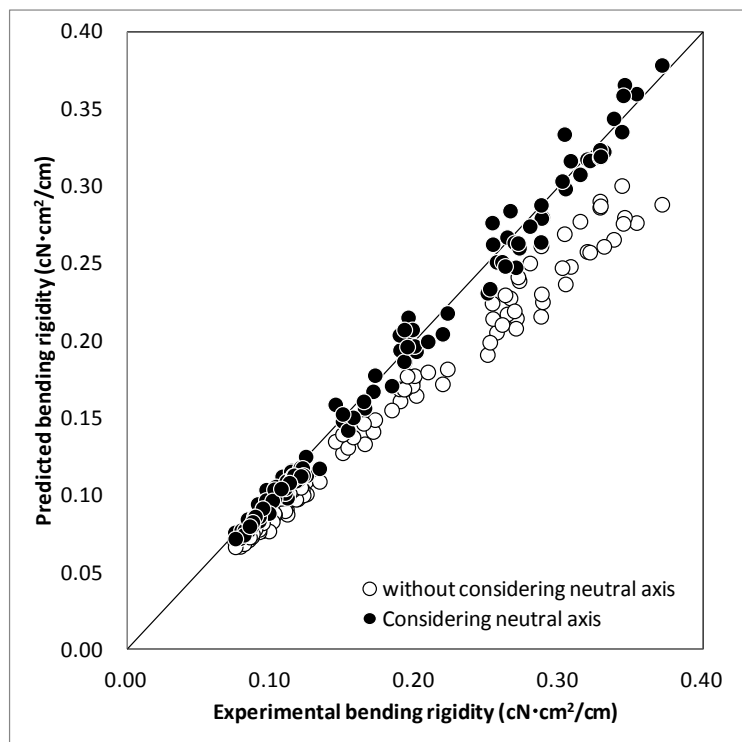

Figure 3 Predicted and experimental bending rigidity of the laminated fabrics by the method with and without considering the position of neutral axis.

Table 8 Mean Absolute Percentage Errors (MAPE) between predicted and experimental bending rigidity with and without considering the position of the neutral axis

\begin{tabular}{ccc}
\hline Laminate condition & \multicolumn{2}{c}{ Mean Absolute Percentage Errors (\%) } \\
\cline { 2 - 3 } & $\begin{array}{c}\text { Method with considering } \\
\text { neutral axis }\end{array}$ & $\begin{array}{c}\text { Method without considering } \\
\text { neutral axis }\end{array}$ \\
\hline S1-all interlining(warp) & 4.0 & 16.2 \\
S1-all interlining(weft) & 4.5 & 11.8 \\
S2-all interlining(warp) & 4.7 & 19.2 \\
S2-all interlining(wet) & 3.9 & 13.9 \\
S3-all interlining(warp) & 6.3 & 11.5 \\
S3-all interlining(weft) & 3.6 & 10.5 \\
S4-all interlining(warp) & 3.4 & 9.7 \\
S4-all interlining(weft) & 2.8 & 11.4 \\
S5-all interlining(warp) & 3.1 & 17.5 \\
S5-all interlining(weft) & 3.5 & 12.8 \\
S6-all interlining(warp) & 1.2 & 9.9 \\
S6-all interlining(weft) & 2.3 & 8.4 \\
\hline All face fabric-all & 3.6 & 12.7 \\
interlinings & & \\
\hline
\end{tabular}


Therefore, it is certain that the bending rigidity of laminated fabric is affected by the position of neutral axes of components. This is equivalent to differentiating between the tensile and compressive moduli in bending of the fabrics. The difference of the moduli was successfully considered in the proposed method. Thus, if the position of the neutral axis of a fabric is obtained, then the bending rigidity of the composite with the fabric and another adhesive interlining can be predicted. With this new method, the bending rigidity of a laminated fabric can be predicted more precisely.

In this study, the satin fabrics were mainly used as samples which show the large prediction errors (over about MAPE 10\%) by the method without considering the position of the neutral axis. However, it should be noted that no all satin fabric will show large prediction errors by the prediction method without considering the neutral axis. The tensile and in-plane compressive moduli of a fabric in bending may be affected by yarn properties in addition to weave structure of a fabric. Therefore, the position of neutral axis may vary in the case of fabric even with the same satin structure.

\section{Conclusion}

A new theory of bending rigidity of laminated fabric was proposed. The position of neutral axis in bending for face fabric is considered in addition to the tensile and in-plane compressive moduli of components.

The proposed method was verified by calculating the bending rigidity of laminated fabrics especially with the samples which bending rigidity cannot be predicted precisely with the method without considering the position of neutral axis. As a result, the relative position of the neutral axis of a face fabric was able to be obtained with the proposed method. The obtained neutral axis of the face fabric did not lie close to the centroid. Using the position of the neutral axis, bending rigidity of laminated fabric was predicted. The predicted bending rigidities showed closer agreement with the experimental data than those by method without considering the position of the neutral axis.

Thus, in the proposed theory, it became clear that the obtained position of the neutral axis in a fabric is reasonably valid and it is able to predict the bending rigidity of laminated fabric more precisely with the position of neutral axis.

Until now, the selection of adhesive interlining was carried out based on experiments and previous data. If the data concerning adhesive interlinings and face fabrics has been compiled once, the prediction of the performance of laminated fabrics made of different combination will be possible. Therefore, this new method will help designers and manufacturers to select suitable adhesive interlinings for garments without extra cost and time.

\section{Appendix}

Notations:

Component 1 adhesive interlining

Component2 face fabric

$B_{1}$ bending rigidity per unit breadth of component1

$B_{2} \quad$ bending rigidity per unit breadth of component2

$B_{12}$ bending rigidity per unit breadth of laminated fabric with component1and component 2

$h_{1} \quad$ thickness of component 1

$h_{2}$ thickness of component2

$R$ radius of curvature

$\eta \quad$ distance from the neutral axis

0 neutral axis of a laminated fabric

$y_{0} \quad$ coordinate of bottom of laminated fabric

$y_{1} \quad$ coordinate of the border of components

$y_{2} \quad$ coordinate of boundary at the top of laminated fabric

$T_{1 C}$ apparent in-plane compressive modulus for longitudinal strain of neutral axis for component1

$T_{2 C}$ apparent in-plane compressive modulus for longitudinal strain of neutral axis for component 2

$T_{1 T}$ apparent tensile modulus for longitudinal strain of neutral axis for component 1

$T_{2 T}$ apparent tensile modulus for longitudinal strain of neutral axis for component 2

$\varepsilon_{1} \quad$ compressive strain at the neutral axis of component 1

$\sigma_{1} \quad$ stress in component1

$F_{1} \quad$ total force of component 1

$F_{2} \quad$ total force of component2

$M_{1} \quad$ couple per unit breadth and unit curvature of component1

$M_{2} \quad$ couple per unit breadth and unit curvature of component2

$E_{1 T}$ elastic modulus of strain in tension of component1

$E_{1 C}$ elastic modulus of strain in compression of component 1

$E_{2 T}$ elastic modulus of strain in tension of component2

$E_{2 C}$ elastic modulus of strain in bending of component 2

$y_{1 n 0}$ distance from the bottom to the neutral axis of component 1

$y_{2 n 0}$ distance from the bottom to the neutral axis of component2 
$Y_{1}$ relative position of neutral axis of component1

$Y_{2}$ relative position of neutral axis of component2

$E_{B T}$ tensile modulus of a fabric

$E_{B C}$ in-plane compressive modulus of a fabric

$b \quad$ breadth

$y_{1 n} \quad$ original neutral axis of component 1

$y_{2 n} \quad$ original neutral axis of component 2

$Y, Y^{\prime}$ relative position of neutral axis of a fabric

\section{$<$ References>}

1. Shishoo R, Klevermar P H, Cednas M, and Olofsson B (1971) Multilayer Textile Structure. Textile Research Journal 41:669-679.

2. Fan J, Leeuwner W, and Hunter L (1997) Compatibility of Outer and Fusible Interlining Fabrics in Tailored Garments part 2 : Relationship between mechanical properties of fused composites and those of outer and fusible interlining fabrics Textile Research Journal 67(3):194-197.

3. Kanayama M and Niwa M (1981) Bending properties of composite fabrics reinforced with fusible interlinings. $J$. Textile Machinery Soc Japan 35(1): 102-112.

4. Kanayama M and Niwa M (1984) Theoretical calculation of bending rigidity of fused fabric composite. J. Textile Machinery Soc Japan 37(10): T161-T167.

5. Kim KO, Inui S, and Takatera M (2011) Verification of Prediction for Bending Rigidity of Woven Fabric Laminated with Interlining by Adhesive Bonding, Textile Research Journal 81(6): 598-607.

6. Dawes V H and Owen J D (1972) The handle and bending behavior of fabric laminates J.Text. Inst. 63: T446-474.

7. Kim KO, Inui S, and Takatera M (2012) Prediction of bending rigidity for laminated fabric with adhesive interlining by a laminate model considering tensile and in-plane compressive moduli Textile Research Journal 82(4): 385-399.

8. Nakura M and Mizutani M (1988) On the position of the neutral plane in the woven fabrics to which the pure bending is applied Journal of the Japan Research Association for Textile End-Uses 29(5): 193-198

9. Lindberg J, Waesterberg L and Svenson R (1960) Wool Fabrics As Garment Construction Materials, J. Text. Inst. 51: T1475-T1493

10. Dahlberg B (1961) Part II: Buckling Textile Research Journal 31(2): 94-99

11. Kawabata S (1980)The standardization and analysis of hand evaluation 2nd ed.: Textile Machinery society of Japan: Osaka 